\title{
Dwarf galaxy candidates found on the SERC EJ sky survey
}

\author{
I.D. Karachentsev ${ }^{1}$, V.E. Karachentseva ${ }^{2}$, A.A. Suchkov ${ }^{3}$, and E.K. Grebel ${ }^{4,5, \star}$ \\ 1 Special Astrophysical Observatory, Russian Academy of Sciences, N. Arkhyz, KChR, 369167, Russia \\ 2 Astronomical Observatory of Kiev University 04053, Observatorna 3, Kiev, Ukraine \\ 3 Space Telescope Science Institute, 3700 San Martin Drive, Baltimore, MD 21218, U.S.A. \\ 4 Department of Astronomy, University of Washington, Box 351580, Seattle, WA 98195-1580, U.S.A. \\ 5 Max Planck Institute for Astronomy, Königstuhl 17, D-69117, Heidelberg, Germany
}

Received May 19; accepted June 17, 2000

\begin{abstract}
We inspected a sky region with declination range of $\left[0^{\circ},-18^{\circ}\right]$ based on the SERC EJ plate copies. As a result a list of 50 nearby dwarf galaxy candidates with angular diameters $\geq 0.5$ is presented. Most of the galaxies have low and very low surface brightness. More than $60 \%$ of the objects are detected for the first time.
\end{abstract}

Key words: dwarf galaxies

\section{Introduction}

Low surface brightness (LSB) dwarf galaxies are believed to be the most numerous galaxy population in the Universe. They are recognized as ideal laboratories to study star formation in different types of galaxies, the evolution of the interstellar medium, and the amount and nature of galactic dark matter; they also represent a key element in studies of galaxy formation and evolution in general. LSB dwarf galaxies are difficult to detect because their surface brightness is typically only a few percent above the dark night sky. For this reason their total number remains unknown even within the Local Group boundaries, not to mention the Local Supercluster and beyond. Some authors believe that $\sim 90 \%$ of all galaxies have surface brightness below the currently achieved detection limit (Disney \& Phillips 1983; McGaugh 1996).

Systematic all-sky searches for LSB dwarfs began after the public distribution of the first photographic survey of the Northern sky (= POSS-I), which covers a sky area at declinations $D>-30^{\circ}$ (van den Bergh 1959, 1966; Karachentseva 1968, 1972, 1973). Also many dwarf galaxies were found when general galaxy catalogues, such as MCG (Vorontsov-Velyaminov et al. 1962), UGC (Nilson

\footnotetext{
* Hubble Fellow.
}

1973), UGCA (Nilson 1974) were compiled based on the POSS-I.

A special search for LSB dwarfs in the southern sky has been conducted by Feitzinger \& Galinsky (1985) on the ESO/SERC sky survey covering a sky area below $-18^{\circ}$. A large number of LSB dwarfs entered in the catalogues by Lauberts (1982) and Arp \& Madore (1987) are based on that survey. Note that due to the better quality of photographic material used for the ESO/SERC survey, one may detect objects that are fainter and have lower surface brightness than those achievable with the POSS-I.

Different lists of LSB dwarf galaxies compiled before 1987 were reinspected by Karachentseva \& Sharina (1988). After re-inspection of several thousand objects presented in different catalogues and lists, they compiled a catalogue of about 1500 LSB dwarf galaxies. The catalogue covers all the sky and has a characteristic depth of $\sim 20 \mathrm{Mpc}$ matching the Local Supercluster boundaries.

\section{New steps in search for dwarf galaxy candidates}

The Second Palomar Sky Survey (=POSS-II) covering the northern hemisphere, $\left[0^{\circ}<D<+90^{\circ}\right]$, together with the SERC Equatorial survey, $\left[0^{\circ}<D<-18^{\circ}\right]$, and the ESO/SERC survey, $\left[-90^{\circ}<D<-18^{\circ}\right]$, provide a unique opportunity to search systematically for LSB dwarfs on the homogeneous photographic material over the entire sky. This has already resulted in a recent discovery of the Andromeda dwarf satellites (Armandroff et al. 1998; Armandroff et al. 1999; Karachentsev \& Karachentseva 1999), and the Local Group dwarf galaxy Cetus (Whiting et al. 1999).

One of the tasks to be performed on the new sky surveys has been a compilation of a complete and representative volume-limited sample of galaxies. To this end Karachentseva \& Karachentsev (2000) have undertaken a search on the POSS-II and the ESO/SERC 
Table 1. List of new dwarf galaxy candidates found in the SERC EJ zone

\begin{tabular}{|c|c|c|c|c|c|c|c|}
\hline No & RA (1950.0) Dec. & \multicolumn{2}{|c|}{$a \times b$} & Type & $\mathrm{SB}$ & NED ident. & Notes \\
\hline 1 & 2 & \multicolumn{2}{|c|}{3} & 4 & 5 & 6 & 7 \\
\hline 1 & $00^{\mathrm{h}} 23^{\mathrm{m}} 38^{\mathrm{s}} .5-11^{\circ} 19^{\prime} 50^{\prime \prime}$ & $5 ! 0$ & $4^{\prime} \cdot 3$ & Sph & $\mathrm{VL}$ & Cetus & LG member [1] \\
\hline 2 & $003744.6-180604$ & 0.9 & 0.6 & $\mathrm{Sph} / \mathrm{Ir}$ & $\mathrm{L}$ & Scu 26 & granulated \\
\hline 3 & $013216.7-073638$ & 0.6 & 0.4 & Ir & $\mathrm{L}$ & $\operatorname{kdg} 7$ & N615 $5^{\prime} \mathrm{NE}[2]$ \\
\hline 4 & $023919.2-083654$ & 1.2 & 1.1 & Sph & $\mathrm{L}$ & & in N1052 gr.? \\
\hline 5 & $024703.0-132506$ & 1.0 & 0.5 & Ir & $\mathrm{L}$ & & \\
\hline 6 & $042759.2-141856$ & 0.9 & 0.4 & Ir & $\mathrm{L}$ & & \\
\hline 7 & $055702.8-130528$ & 0.8 & 0.2 & Ir & $\mathrm{L}$ & & distant? \\
\hline 8 & $064215.2-175251$ & 1.8 & 0.3 & Ir & $\mathrm{H}$ & IC 2171 & different $V_{\mathrm{h}}[3]$ \\
\hline 9 & $064444.3-175310$ & 0.5 & 0.35 & Epec & $\mathrm{H}$ & CGMW 1-0381 & $V_{\mathrm{h}}=696$ \\
\hline 10 & $071028.9-074412$ & 1.0 & 0.25 & Ir & $\mathrm{L}$ & & \\
\hline 11 & $072841.0-005204$ & 0.7 & 0.4 & Ir & $\mathrm{L}$ & & distant? \\
\hline 12 & $085105.8-174742$ & 0.5 & 0.4 & Ir & $\mathrm{L}$ & & patchy \\
\hline 13 & $093305.4-160634$ & 1.2 & 0.2 & Ir & $\mathrm{VL}$ & VC,94 & 4 gal. $\sim 7^{\prime} \mathrm{SW}$ \\
\hline 14 & $093755.0-033930$ & 1.1 & 1.0 & Ir & $\mathrm{L}$ & PGC 27612 & \\
\hline 15 & $095240.9-060200$ & 1.3 & 0.3 & Ir? & $\mathrm{H}$ & APMUKS & comp. N3115? \\
\hline 16 & $095718.2-090639$ & 0.9 & 0.7 & Ir? & $\mathrm{VL}$ & & comp. N3115? \\
\hline 17 & $095909.4-080027$ & 1.4 & 0.25 & Ir & $\mathrm{H}$ & MCG-1-26-11 & comp. N3115? \\
\hline 18 & $100312.3-074416$ & 1.7 & 1.4 & S0pec & $\mathrm{H}$ & NGC $3115 \mathrm{dw} 1$ & $V_{\mathrm{h}}=698$ \\
\hline 19 & $102200.5-121043$ & 0.7 & 0.6 & $\operatorname{Ir}$ & $\mathrm{L}$ & & \\
\hline 20 & $110206.3+001940$ & 0.5 & 0.4 & $\mathrm{Ir} / \mathrm{Sph}$ & $\mathrm{L}$ & APMUKS & comp. N3521? \\
\hline 21 & $110301.7-013536$ & 1.7 & 0.8 & Ir & $\mathrm{L}$ & UGC 6145 & $V_{\mathrm{h}}=740$, c. N $3521 ?$ \\
\hline 22 & $110334.5-011036$ & 0.5 & 0.4 & Ir? & $\mathrm{L}$ & APMUKS & comp. N3521? \\
\hline 23 & $110342.2-140804$ & 3.5 & 1.2 & Ir & $\mathrm{L}$ & & \\
\hline 24 & $111120.3-031531$ & 0.5 & 0.4 & Ir & $\mathrm{L}$ & APMUKS & \\
\hline 25 & $114245.5-165946$ & 1.2 & 0.7 & Ir & $\mathrm{VL}$ & & v. blue \\
\hline 26 & $115118.3-144451$ & 1.8 & 1.1 & Ir & $\mathrm{VL}$ & & v. blue \\
\hline 27 & $121930.9-093123$ & 0.8 & 0.3 & Ir & $\mathrm{L}$ & & distant? \\
\hline 28 & $122122.0-144034$ & 1.2 & 1.0 & Ir & EL & & \\
\hline 29 & $123438.4-101321$ & 1.4 & 0.6 & Ir & $\mathrm{L}$ & PGC 42120 & comp. N4594? \\
\hline 30 & $123500.5-083532$ & 1.0 & 0.5 & $\mathrm{Ir}$ & $\mathrm{L}$ & & comp. N4594? \\
\hline 31 & $123558.0-101256$ & 0.8 & 0.7 & Sph & $\mathrm{VL}$ & & comp. N4594? \\
\hline 32 & $123718.3-112835$ & 0.6 & 0.5 & Sph & EL & & comp. N4594? \\
\hline 33 & $123732.7-120525$ & 0.5 & 0.4 & Sph & $\mathrm{VL}$ & & comp. N4594? \\
\hline 34 & $123842.7-113912$ & 0.8 & 0.7 & Sph & $\mathrm{VL}$ & & comp. N4594? \\
\hline 35 & $123958.6-144002$ & 0.4 & 0.4 & Ir & EL & & bluish \\
\hline 36 & $124402.8-034810$ & 0.9 & 0.65 & Ir & $\mathrm{L}$ & $\operatorname{kdg} 198$ & \\
\hline 37 & $124524.2-122300$ & 0.6 & 0.5 & Sph? & $\mathrm{VL}$ & & \\
\hline 38 & $125056.6-053925$ & 1.2 & 0.5 & Ir & $\mathrm{L}$ & APMUKS & \\
\hline 39 & $125106.5-054846$ & 0.8 & 0.6 & Sph & EL & & \\
\hline 40 & $125715.7-135104$ & 1.0 & 0.6 & Ir & $\mathrm{VL}$ & & \\
\hline 41 & $130307.6-072929$ & 1.4 & 1.1 & Ir & $\mathrm{L}$ & $\operatorname{kdg} 218$ & comp. N4948? \\
\hline 42 & $130342.7-074931$ & 1.2 & 0.9 & Ir & $\mathrm{VL}$ & & comp. N4948? \\
\hline 43 & $130639.6-170555$ & 0.7 & 0.5 & $\mathrm{Ir} ?$ & EL & & \\
\hline 44 & $133023.5-120020$ & 0.5 & 0.3 & Ir & $\mathrm{L}$ & & blue; distant? \\
\hline 45 & $141106.0-015712$ & 0.6 & 0.4 & Sph? & $\mathrm{VL}$ & $\operatorname{kdg} 230$ & distant? \\
\hline 46 & $142547.0-084155$ & 0.5 & 0.5 & Ir & $\mathrm{L}$ & & distant? \\
\hline 47 & $143238.5-165643$ & 1.8 & 0.9 & Ir & $\mathrm{L}$ & & \\
\hline 48 & $160302.4-042616$ & 1.3 & 1.0 & Ir & $\mathrm{L}$ & & distant S? \\
\hline 49 & $164045.7-075800$ & 1.1 & 0.4 & Ir & $\mathrm{L}$ & & distant? \\
\hline 50 & $173900.1-043559$ & 1.3 & 0.5 & Ir & $\mathrm{L}$ & & distant S? \\
\hline
\end{tabular}

[1] Discovered by Whiting et al. (1999).

[2] Probably belongs to a group of NGC 584, 596, 600, 615 with the mean $V_{\mathrm{h}}=1850 \mathrm{~km} \mathrm{~s}^{-1}$.

[3] There are two different velocity estimates: $V_{\mathrm{h}}=3395 \mathrm{~km} \mathrm{~s}^{-1}$.

(Visvanathan \& Yamada 1996), and $V_{\mathrm{h}}=784 \mathrm{~km} \mathrm{~s}^{-1}$ (Theureau et al. 1998). 


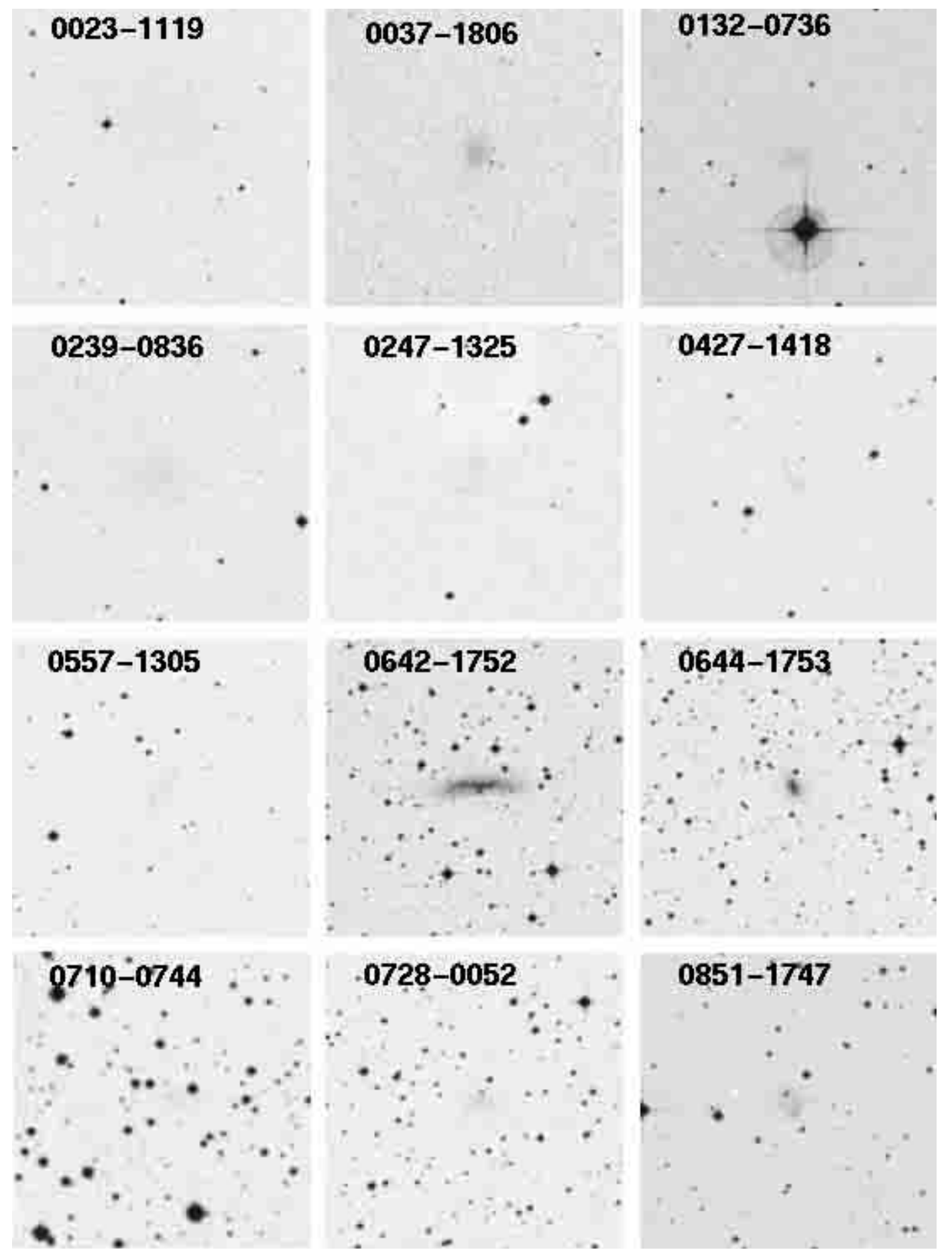

Fig. 1. Digital Sky Survey $5^{\prime} \times 5^{\prime}$ images of 50 dwarf galaxy candidates (continued in Figs. 2-5). North is up, and East is to the left

copies for LSB dwarf galaxy candidates with angular diameters $a \geq 0.5$ around all the Local Volume $(=\mathrm{LV})$ galaxies. The LV galaxies with corrected radial velocities $V_{0}<500 \mathrm{~km} \mathrm{~s}^{-1}$ were taken from the list by Karachentsev (1994), which is an up-dated version of Kraan-Korteweg \& Tammann's (1979) and Schmidt \& Boller's (1992) catalogues of nearby galaxies. Altogether 260 nearby dwarf galaxy candidates have been found (henceforth referred to as kk-objects or kk-galaxies), more than a half of them previously uncatalogued. 


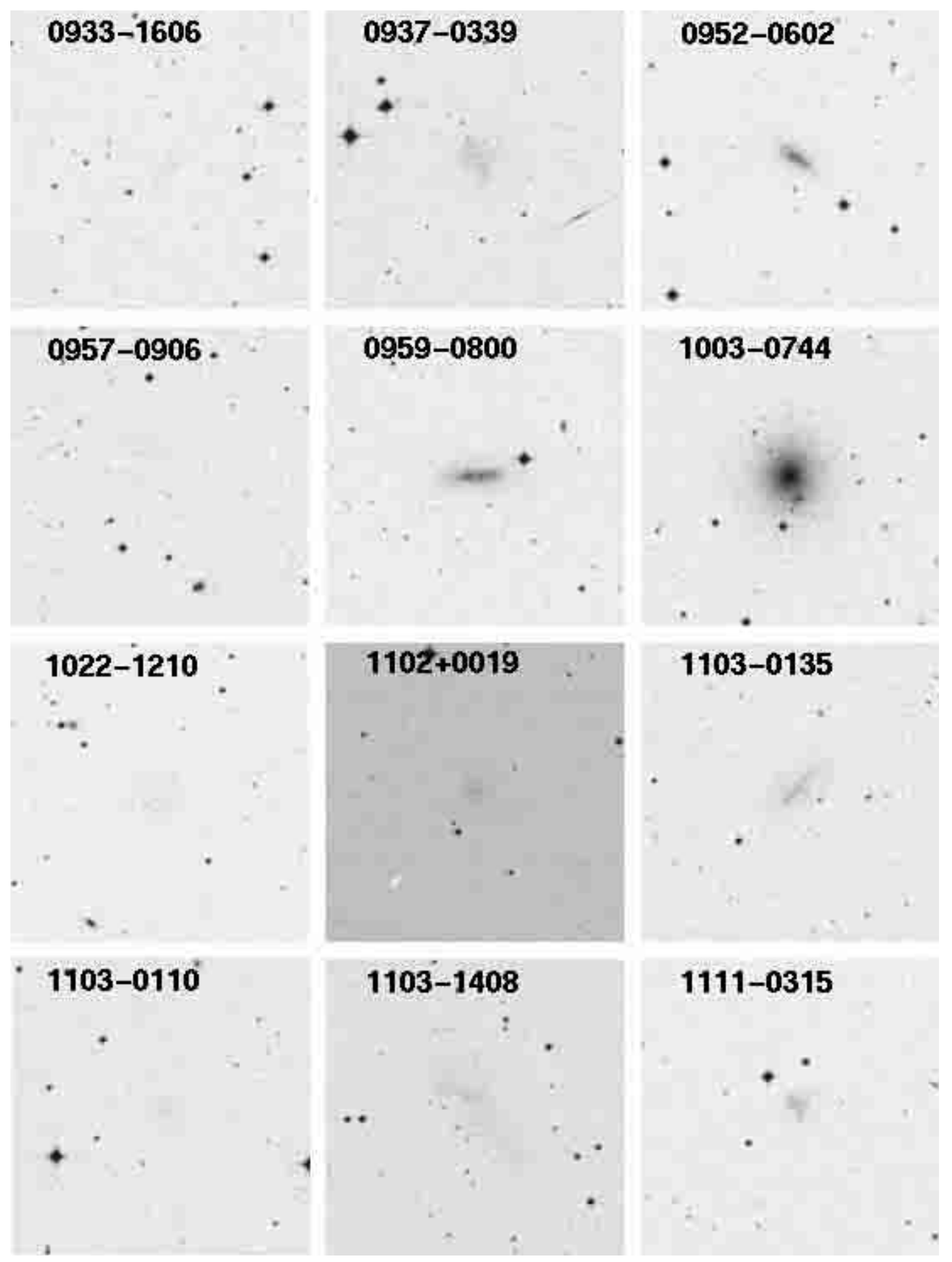

Fig. 2. Digital Sky Survey images of 50 dwarf galaxy candidates (continued from Fig. 1) 


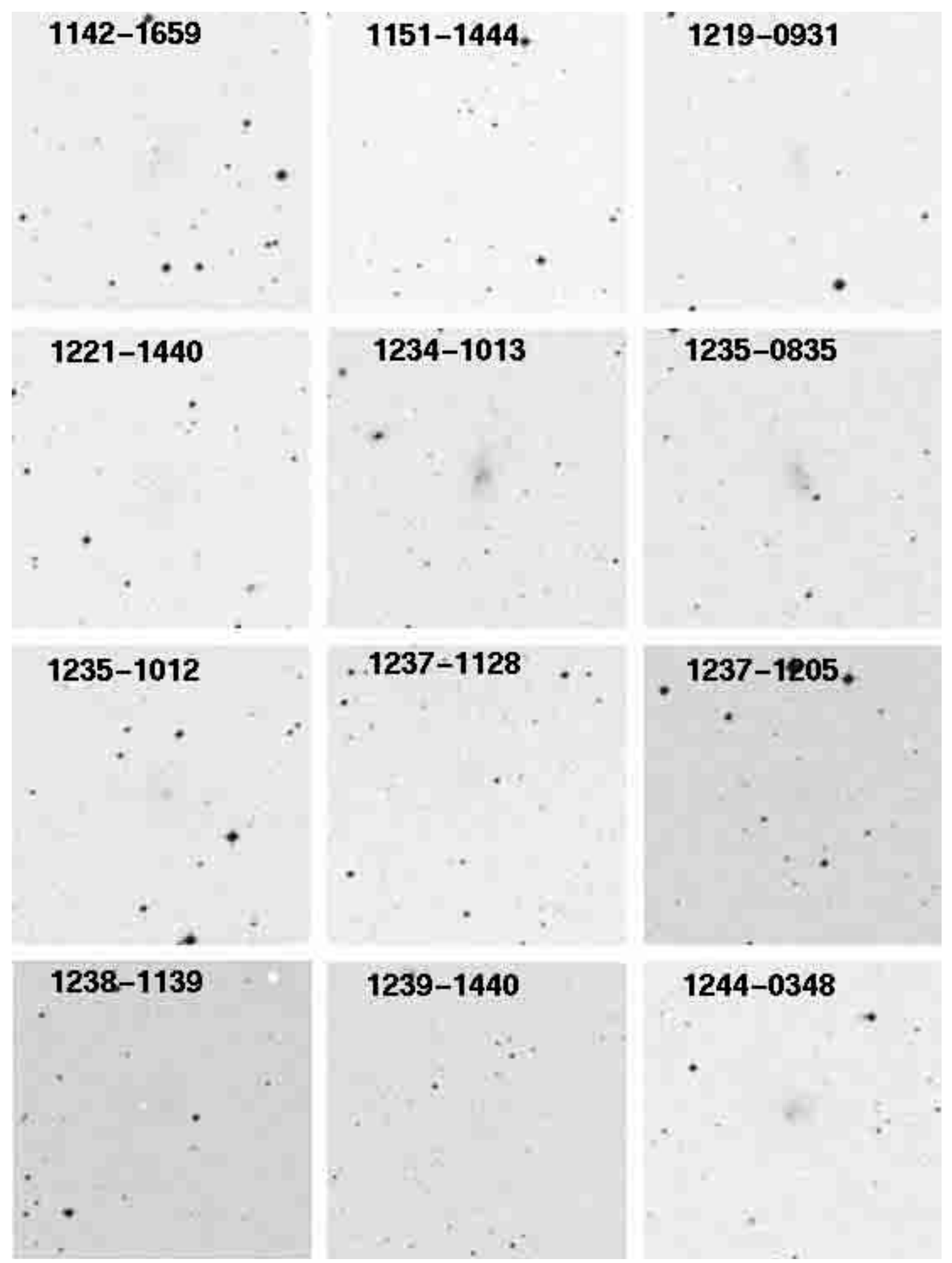

Fig. 3. Digital Sky Survey images of 50 dwarf galaxy candidates (continued from Fig. 1) 


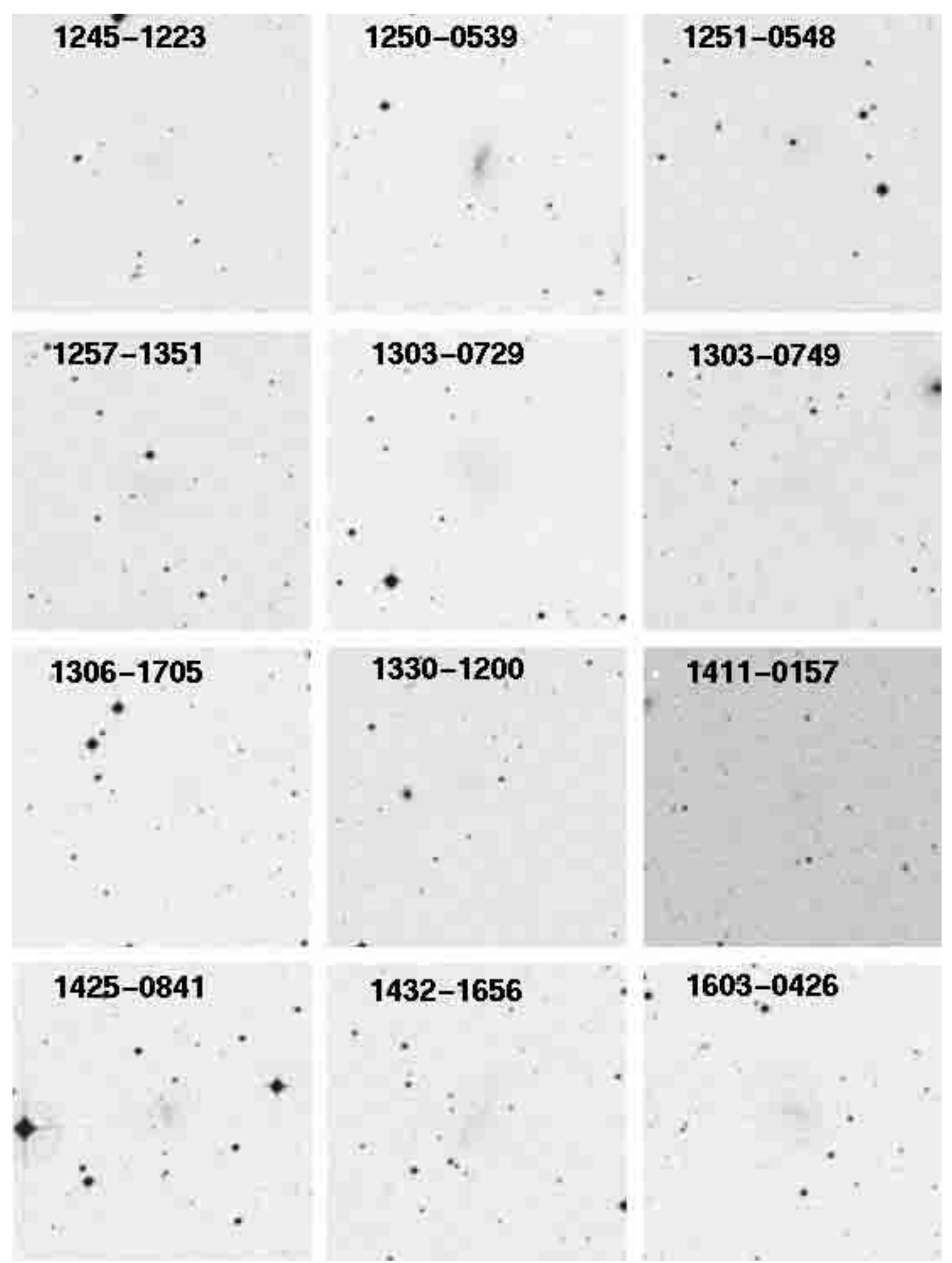

Fig. 4. Digital Sky Survey images of 50 dwarf galaxy candidates (continued from Fig. 1) 

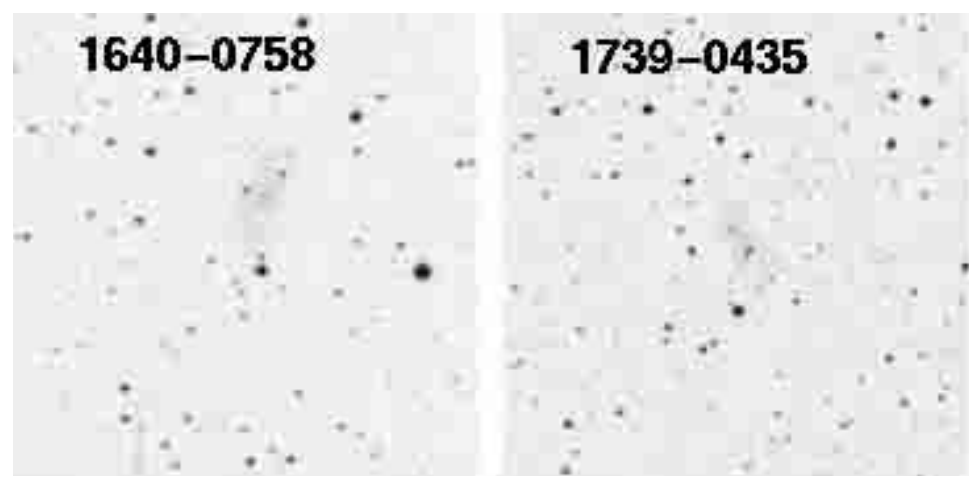

Fig. 5. Digital Sky Survey images of 50 dwarf galaxy candidates (continued from Fig. 1)

HI observations of the kk-objects with the 100-m Effelsberg radio telescope (Huchtmeier et al. 1997, 2000) have shown that:

1. the HI detection rate is about $60 \%$ (a rather high value taking into account that the kk-list contains a large number of dSphs);

2. the median radial velocity in the sample is about $1200 \mathrm{~km} \mathrm{~s}^{-1}$, indicating that the kk-objects are mainly real dwarf galaxies;

3. there are $40 \mathrm{kk}$-galaxies with $V_{0}<500 \mathrm{~km} \mathrm{~s}^{-1}$, which essentially increases the LV sample.

The next steps involved the following:

1. A search for dwarf galaxy candidates in the Tully Local Void region, covering $\sim 6000 \square^{\circ}$ area around $\left\{\mathrm{RA}=18^{\mathrm{h}} 38^{\mathrm{m}}, D=+18^{\circ}\right\}$, yielded new 78 objects (Karachentseva et al. 1999);

2. An overall search in the ESO/SERC sky region resulted in 81 dwarf galaxy candidates (Karachentseva \& Karachentsev 2000);

3. An overall search on the POSS-II, the northern hemisphere (except the Virgo cluster region and the Local Void area), yielded 101 dwarf galaxy candidates (Karachentsev et al. 2000). Many of them have been observed by W. Huchtmeier with the Effelsberg radio telescope.

\section{A list of dwarf galaxy candidates in the equatorial zone}

The last step of the work has been accomplished on the J, F glass copies of the SERC Equatorial survey belonging to STScI. Altogether 216 both "blue" and "red" $6 \times 6$ degree plates have been inspected in the declination zones -5 , -10 , and $-15^{\circ}$ along with 5 equatorial POSS-II fields absent in our previous inspection. Similar to previous work, we selected dwarf galaxy candidates with angular diameters $\geq ! 5$ by their low or very low surface brightness. Some of the high surface brightness objects were also taken into account.

After exclusion of emulsion defects, isolated Galactic cirrus, and probable distant "normal" LSB galaxies we have compiled the main list of 50 objects. This list is given in Table 1. The table columns contain:

(1) the running number;

(2) equatorial coordinates (epoch 1950.0);

(3) major and minor diameters measured visually on blue plates;

(4) the morphological type in usual designations ("d" for "dwarf" is omitted);

(5) a rough estimate of surface brightness (=SB): $\mathrm{H}$ - high (equal or brighter than the $\mathrm{SB}$ of a normal spiral galaxy, $22-23$ mag $\operatorname{arcsec}^{-2}$ ), L - low $\left(23\right.$ - 24 mag $\left.\operatorname{arcsec}^{-2}\right)$, VL - very low $\left(24-25 \mathrm{mag} \operatorname{arcsec}^{-2}\right), \mathrm{EL}$ - extremely low $\left(25-26\right.$ mag $\left.\operatorname{arcsec}^{-2}\right)$;

(6) galaxy name in other catalogues and lists as given in the NASA Extragalactic Database (=NED);

(7) comments concerning morphology, galaxy membership, etc.

Note, that the object \#1 (Cetus) was found by us independently from Whiting et al. (1999). In this paper, which was devoted to the discovery and detailed investigation of the Cetus LG galaxy, the authors reported the finding of 75 new VLSB dwarfs in a sky region at $D<3^{\circ}$ covered by the ESO/SERC and SERC EJ surveys. Unfortunately, we were unable to compare our list of objects with their unpublished list.

Figures $1-5$ displays $5^{\prime} \times 5^{\prime}$ images of 50 dwarf galaxy candidates taken from the Digital Sky Survey.

In Table 2 we give for comparison a list of other 17 LSB objects, which were isolated using the same criterion but exhibited radial velocities in excess of $500 \mathrm{~km} \mathrm{~s}^{-1}$. The table columns provide the same quantities as in Table 1. We added only two columns, with total magnitude, $B_{\mathrm{t}}$, and heliocentric radial velocity, $V_{\mathrm{h}}$, from NED. According to the NED data, all objects in Table 2 were catalogued 
Table 2. Other candidates selected with the same criterion and rejected due to their velocities

\begin{tabular}{|c|c|c|c|c|c|c|c|c|c|}
\hline No & RA (1950.0) Dec. & \multicolumn{2}{|c|}{$a \times b$} & $\mathrm{~T}$ & SB & NED Ident. & $B_{\mathrm{T}}$ & $V_{\mathrm{h}}$ & Notes \\
\hline 51 & $02^{\mathrm{h}} 31^{\mathrm{m}} 28^{\mathrm{s}} .0-06^{\circ} 34^{\prime} 44^{\prime \prime}$ & 0.8 & 0.4 & $\mathrm{Ir}$ & $\mathrm{L}$ & P09774 & - & 1407 & knot \\
\hline 52 & $024650.9-025143$ & 0.9 & 0.5 & Ir? & $\mathrm{L}$ & UGC A44 & - & 1094 & \\
\hline 53 & $040639.3-084528$ & 1.0 & 0.6 & Ir & $\mathrm{L}$ & P14562 & 一 & 894 & \\
\hline 54 & $053936.7-123509$ & 1.0 & 0.4 & $\mathrm{Sm}$ & $\mathrm{L}$ & P17621 & - & 2245 & \\
\hline 55 & $054755.8-101845$ & 2.0 & 0.9 & $\mathrm{SB} ?$ & $\mathrm{~L}$ & P17965 & - & 895 & \\
\hline 56 & $054944.0-110905$ & 0.9 & 0.8 & $\mathrm{Ir} ?$ & $\mathrm{~L}$ & P18027 & 15.5 & 903 & pair w.\#55 \\
\hline 57 & $084104.4-171215$ & 1.8 & 0.5 & Ir & $\mathrm{L}$ & P24494 & - & 2019 & knots \\
\hline 58 & $105029.1+024533$ & 1.5 & 0.5 & Ir & $\mathrm{L}$ & L135768 & 17.4 & 1054 & \\
\hline 59 & $111013.9-001739$ & 0.5 & 0.4 & Ir & $\mathrm{L}$ & ISI, 96 & 17.8 & 8472 & patchy, blue \\
\hline 60 & $122035.1-134005$ & 1.4 & 0.9 & Ir & $\mathrm{L}$ & UGC A278 & - & 1155 & knots \\
\hline 61 & $124218.7-085115$ & 4.0 & 1.8 & $\mathrm{Sm}$ & $\mathrm{L}$ & UGC A295 & - & 1378 & \\
\hline 62 & $125629.6-115731$ & 1.2 & 0.8 & Ir & $\mathrm{L}$ & UGC A312 & - & 1307 & \\
\hline 63 & $132343.5+024306$ & 0.8 & 0.4 & $\mathrm{Ir}$ & $\mathrm{L}$ & ISI, 96 & 16.8 & 1137 & blue \\
\hline 64 & $132539.4+023220$ & 0.8 & 0.45 & Ir & $\mathrm{L}$ & ISI, 96 & 16.3 & 1221 & $\sigma_{v}=275$ \\
\hline 65 & $141147.0-024759$ & 0.8 & 0.25 & Ir & $\mathrm{L}$ & ISI, 96 & 15.8 & 1853 & $\sigma_{v}=275$ \\
\hline 66 & $143120.7+014214$ & 1.0 & 0.2 & Ir & $\mathrm{L}$ & ISI, 96 & 17.4 & 1829 & \\
\hline 67 & $200641.4-062605$ & 2.2 & 1.4 & $\mathrm{~S}$ & $\mathrm{~L}$ & UGC A417 & - & 1425 & \\
\hline
\end{tabular}

ISI, $96=$ Impey et al. (1996).

earlier, and their radial velocities together with their angular extent and low surface brightness confirm that these are dwarf galaxies.

\section{Concluding remarks}

In the sky region covered by the SERC Equatorial survey, we have found 50 low and very low surface brightness objects. Most of them presumably are nearby dwarf galaxies. More than half of these objects were previously uncatalogued. The presented results increase the number of possible dwarf companions around the nearby giant galaxies NGC 3115 and NGC 4594.

This work completes the entire-sky search for nearby LSB dwarf galaxy candidates. A total of $\sim 600$ objects have been found, half of them for the first time. The HI observations of these objects carried out by W. Huchtmeier confirm that most of them are really dwarf galaxies located in the Local Supercluster.

Acknowledgements. We wish to thank the anonymous referee for comments/suggestions that helped to improve the paper. IDK and VEK are grateful to STScI for the support of this collaborative project through the Science Visitor Program. We are also grateful to Dr. Conrad Sturch who provided us with all the facilities necessary to accomplish this work. IDK, VEK, and EKG acknowledge partial support through an Henri Chrétien International Research Grant administered by the American Astronomical Society. EKG acknowledges support by NASA through grant HF-01108.01-98A from the Space Telescope Science Institute. In the present work we used photographic data obtained with the UK Schmidt Telescope. The UK Schmidt Telescope was operated by the Royal Observatory Edinburgh, with funding from the UK Science and Engineering Research Council (later the UK Particle Physics and Astronomy Research Council), until 1988 June, and thereafter by the Anglo-Australian Observatory. This work has made use of the NASA/IPAC Extragalactic Database (NED) which is operated by the Jet Propulsion Laboratory, California Institute of Technology, under contract with NASA. The Digitized Sky Surveys were produced at the Space Telescope Science Institute under U.S. Government grant NAG W-2166.

\section{References}

Armandroff T.E., Davies J.E., Jacoby G.H., 1998, AJ 116, 2287 Armandroff T.E., Jacoby G.H., Davies J.E., 1999, AJ 118, 1220 Arp H.C., Madore B.F., 1987, A Catalogue of southern peculiar galaxies and associations. Cambridge University Press, I, II

Disney M., Phillips S., 1983, MNRAS 205, 563

Feitzinger J.W., Galinski T., 1985, A\&AS 61, 503

Huchtmeier W.K., Karachentsev I.D., Karachentseva V.E., 1997, A\&A 322, 375

Huchtmeier W.K., Karachentsev I.D., Karachentseva V.E., Ehle M., 2000, A\&AS 141, 469

Impey C.D., Sprayberry D., Irwin M.J., Bothun G.D., 1996, ApJS 105, 209 (ISI)

Karachentsev I.D., 1994, A\&Ap Trans. 6, 1

Karachentsev I.D., Karachentseva V.E., 1999, A\&A 141, 469 
Karachentsev I.D., Karachentseva V.E., Huchtmeier W.K., 2000 (in preparation)

Karachentseva V.E., 1968, Soobsh. Byurakan. Obs. 39, 62

Karachentseva V.E., 1972, Astron. Tzirk. 723, 1

Karachentseva V.E., 1973, Astrophyz. Issled. (Izv. SAO) 5, 10

Karachentseva V.E., Sharina M.E., 1988, Catalogue of low surface brightness dwarf galaxies, Comm. Spec. Astrophys. Obs. 57, 3

Karachentseva V.E., Karachentsev I.D., Richter G.M., 1999, A\&AS 135, 221

Karachentseva V.E., Karachentsev I.D., 2000, A\&AS (accepted)

Kraan-Korteweg R.C., Tammann G.A., 1979, Astron. Nachr. 300,181

Lauberts A., 1982, ESO/Uppsala Survey of the ESO(B) Atlas. Garching, European Southern Observatory (ESO)

McGaugh S.S., 1996, MNRAS 280, 337
Nilson P., 1973, Uppsala General Catalogue of Galaxies, Uppsala Astron. Obs. Ann. 6 (UGC)

Nilson P., 1974, Catalogue of Selected Non-UGC Galaxies, Uppsala Astron. Obs. Rep. 5

Schmidt K.-H., Boller T., 1992, Astron. Nachr. 313, 189

Theureau G., Bottinelli L., Coudreau-Durand N.,

Gouguenheim L., Hallet N., Loulergue M., Paturel G., Teerikorpi P., 1998, A\&AS 130, 333

van den Bergh S., 1959, The Catalogue of dwarf galaxies, Publ. D.D.O. v. II, No. 5, 147

van den Bergh S., 1966, AJ 71, 33

Visvanathan N., Yamada T., 1996, ApJS 107, 521

Vorontsov-Velyaminov B.A., Krasnogorskaja A.A., Arkhipova V.P., 1962-1968, The Morphological Catalogue of Galaxies. Moscow State Universe, Moscow, I-IV

Whiting A.B., Hau G.K., Irwin M., 1999, AJ 118, 2767 